\title{
Advice for archaeological survey with recent technologies
}

\author{
Andrea Angelini ${ }^{1}$, Damiano Portarena ${ }^{2}$ \\ ${ }^{1}$ Institute for the Technologies Applied to Cultural Heritage, CNR-ITABC, Monterotondo, Italy \\ 2 University of Rome Tor Vergata, Rome, Italy
}

\section{ABSTRACT}

Despite the technologies are now part of the archaeological discipline, they must not divert attention from archaeological issues and should be aimed at solving different historical and methodological questions, such the phases of a monument or innovative representation methods. The paper focuses the attention mainly on the use of range-data and image-based systems applied to the archaeological heritage, in order to highlight differences between the techniques and the related errors. Some considerations are necessary in order to find possible solutions for improving the quality and the accuracy of a survey project, considering the recent and innovative techniques adopted in archaeology. At the same time the authors provided, where possible, some solutions and suggestions for reducing the errors and checking the general quality of the work. Different experimentations have been made on some case studies that show how to manage technologies trying to reduce as much as possible the errors in the different phases of the survey pipeline. A specific part has been dedicated to the photogrammetric process from drones compared to traditional acquisitions, usually performed with aluminium poles, and the common errors in the representation of the archaeological excavations.

Section: RESEARCH PAPER

Keywords: Archaeological Survey; Laser Scanner, Photomodeling, Drones, Surface Reconstruction, Texture Mapping

Citation: Andrea Angelini, Damiano Portarena, Advice for archaeological survey with recent technologies, Acta IMEKO, vol. 7, no. 3, article 8, October 2018, identifier: IMEKO-ACTA-07 (2018)-03-08

Section Editor: Egidio De Benedetto, University of Salento, Italy

Received April 13, 2018; In final form September 21, 2018; Published October 2018

Copyright: (C) 2018 IMEKO. This is an open-access article distributed under the terms of the Creative Commons Attribution 3.0 License, which permits unrestricted use, distribution, and reproduction in any medium, provided the original author and source are credited

Funding: The methodological work is part of the research activity carried out in the CNR.

Corresponding author: Andrea Angelini, andrea.angelini@itabc.cnr.it

\section{GENERAL INTRODUCTION}

Since the use of direct methods, the survey activity has always represented the base for studying and analyzing ancient monuments. Above all in archaeology, nowadays, it is necessary to deal with new technologies. The recent survey techniques are characterized by user-friendly instruments able to collect large amounts of data. Important for data processing, the survey activity must be a valid support to the archaeological investigation and not the aim of a research project.

For this reason, it is necessary to make some considerations on the appropriate use of these techniques, adjusting the new technologies to the historical-archaeological needs.

Different errors can be added together and influence the final results, so some aspects need to be clarified. The archaeologist should be a professional figure able to move on different application fields and skilled above all in survey acquisition, data processing, synthesis of the results and 3D/2D representation.
The aim of the paper is to highlight the measurement errors that occur during a survey, considering the recent and innovative techniques adopted in archaeology. At the same time the authors provided, where possible, some solutions and suggestions for reducing the errors and checking the general quality of the work [1].

The main survey techniques used in archaeology and architecture are focused mainly on range-data (laser scanning) and image-based systems (photomodeling). Based on completely different geometric principles the two techniques present similar results (point clouds) [2] [3]. The two methods are based on automated processes that do not allow an appropriate data control, like in a "black box" model.

Different output can be extracted from point clouds in order to improve the results. Graphic documentation represents the main instrument for analyzing and studying an archaeological subject and, generally, it is the base for the virtual reconstruction process. 
Some considerations are necessary for evaluating common errors generated during an archaeological survey, in order to find possible solutions and improving the quality and the final accuracy of a project. The paper focuses the attention mainly on the use of laser scanner and digital photogrammetric systems, but it evidences also different issues associated to the processing phase. The latter has been analyzed through the use of integrated software and focused mainly in the surface reconstruction and texture mapping process for the generation of reality-based virtual models.

Some experimentations were performed on different case studies in order to highlight differences between the techniques and the related errors. A specific part was also dedicated to the use of drones (Unmanned Aerial Vehicles, UAVs) in archaeology compared to traditional acquisitions, usually made with aluminium poles, and the errors in the representation of the archaeological excavations.

\section{THE MEASUREMENTS ERRORS IN THE SURVEY}

The aim of a survey project and the choice of the representation scale influence directly the parameters for an appropriate acquisition phase.

In addition to the instrumental error declared by manufacturers, the main element to consider is the error allowed in 2D representation (besides the graphic error). It gives us information about the value beneath which it makes no sense to take measurements. This value was established in relation to the human sight $(0.3 \mathrm{~mm})$, so that in the $1: 50$ representation scale, the maximum allowed error is $1.5 \mathrm{~cm}$ while, for instance, in the 1:100 representation scale the allowed error is $3 \mathrm{~cm} \mathrm{[4].}$

Although the resolution and accuracy of the instruments are

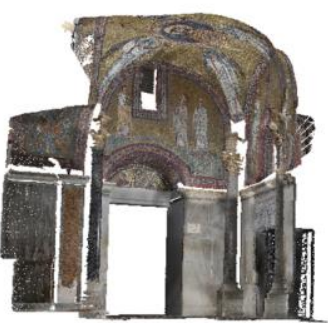

scan 1

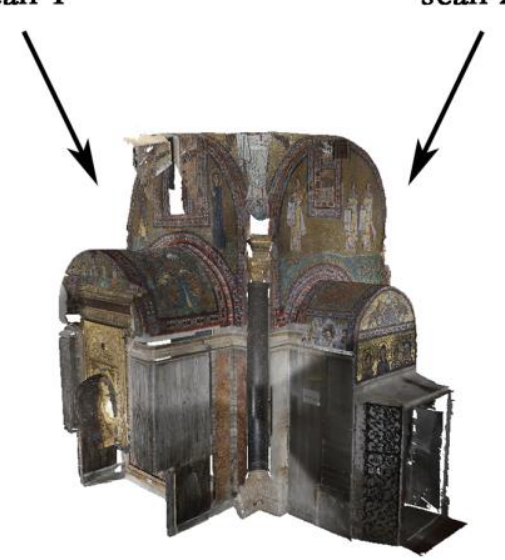

ICP alignment

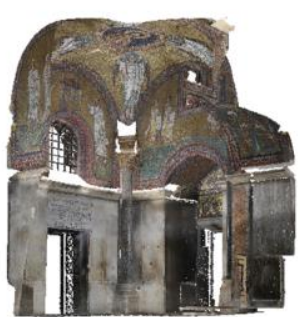

scan 2 very high [5] [6], main errors occur both during the acquisition phase and the processing of the point clouds (registration of the scans), also considering the high number of acquisitions necessary for accomplishing a survey.

The integration of different survey techniques allows to have complete and heterogeneous data in the same reference system but usually it tends to decrease the accuracy of an entire project.

Still today survey methods in archaeology and architecture are based on data integration between laser scanner and topographic systems. With the aid of a total station, traverses are performed in order to register the scans of a survey project and to decrease the spread error of each scan of the laser. The entire scan project is registered through the use of planar targets (more recently spherical targets for automatic registration), the Ground Control Points (GCPs). More precisely, each single scan of the laser presents the same characteristics of the main nodes of a total station, due to the same method of acquisition (polar).

In Topography each node of a traverse suffers from a spread error that must be compensated with known algorithms (angular and distance compensation) [7]. The approach with the GCPs allows to align the scans in the reference system, checking the registration error of the final survey. The MSE (mean squared error) is the average error of the scans based on the GCPs used in the registration phase. The choice of adequate points and their arrangement represent the main quality parameters for the registration step.

Since the $90 \mathrm{~s}$ a series of algorithms for the correct mutual position of the "scans" has been implemented. Such algorithms are known as Iterative Closest Points (ICPs), able to move a point cloud over another point cloud (C2C registration) on the basis of thousands of points (from 5.000 to 20.000 and above), thus reducing the final overlapping error (Figure 1) [8] [9]. The algorithm needs a pre-arranged combination of two scans and oriented points in order to be satisfied. This approach allowed to modify the procedure during the acquisition phase. At present, it is more important to have a good overlap (60-80\%) between the scans in order to satisfy the requirements of the ICP algorithms. The ICP registration is able to reduce the spread error down to a few millimetres, effectively excluding a topographical survey.

One of the risks is that the acquisition of GCPs with a total station could increase the registration value due to systematic errors included both in the acquisition on the field and during the processing step. Once the clouds have been registered,

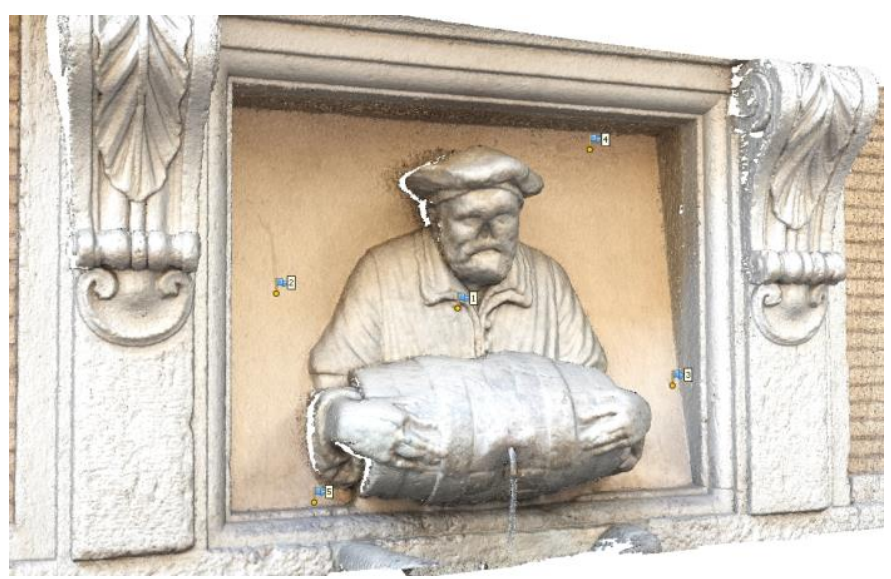

Figure 2. Dense cloud generated from photomodeling system with a $50 \mathrm{~mm}$ optic lens and 5 GCPs identified on the numerical model. algorithms in reliable way.
Figure 1. Two and more point clouds can be aligned according to ICPS 


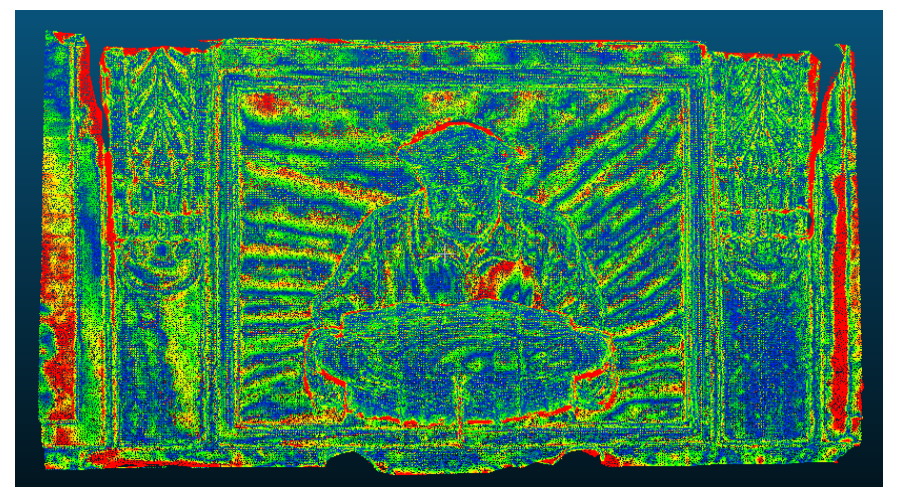

Figure 3. The distance between the two point clouds highlighted different discontinuities in the dense-cloud reconstruction process.

usually the bundle adjustment algorithm is applied to distribute the error on the entire scan project. Below some considerations on ICPs registration method:

- ICPs algorithms are based on statistical methods for the recognition of the points between two clouds. Although the results seem to be accurate, the points control is not available. The target method (GCPs) is based on limited number of points but each error can be controlled any time from the user;

- despite ICPs algorithms are very useful for data integration, they are not able to satisfy all the conditions. Some archaeological subjects need the topography to correct the mutual position of the scans, considering their extension and complexity. The entire fortress wall of an ancient settlement represents an example where the topography cannot be completely excluded. In order to avoid that the walls do not close (assuming the beginning and the end of a closed ideal figure) it is necessary to define a verifiable traverse and specific target for referencing the entire scan project.

In non-urban areas the authors suggest the definition of the main traverse with a differential GPS system (DGPS) instead of a total station, used eventually only for the recognition of the target. DGPS allows to collect information of each node of the traverse with the same instrumental error, influenced only by satellites visibility and disposition, thus avoiding further corrections $( \pm 1 \mathrm{~cm}$ over the entire investigated area. The system used in Real Time mode is able to maintain the same

\section{Histogram of the distances between the two models}

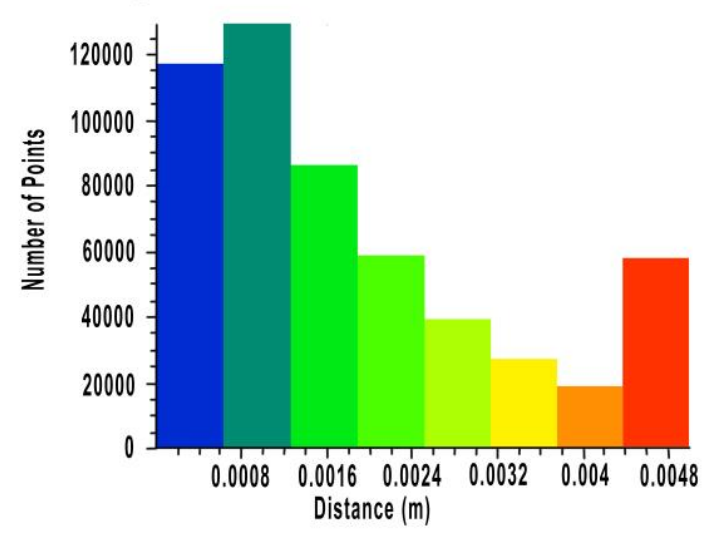

Figure 4 . The histogram shows the 8 classes where points are distributed Only a small part of them is included in the minimum distance between 0 and $0.6 \mathrm{~mm}$. accuracy up to $3-5 \mathrm{Km})[10,11]$. By orienting the total station on 3 nodes of the DGPS traverse it is possible to reference (and georeference) each new job of the scan project.

Similar issues occur also in the photomodeling process. The literature in this field is very wide $[12,13]$, so only some considerations will be presented in relation to the archaeological survey. Different characteristics and procedural errors can sum up together (Figure 2):

- the size of the camera sensor (full-frame, APS-C, the real sensor dimension expressed in $\mathrm{mm}$ varies according to the device);

- the type of lens used (fixed or zoom) and its characteristics expressed by MTF graphic (resolution expressed in lines $/ \mathrm{mm}$ and contrast);

- $\quad$ the maximum resolution of the camera (MP);

- the calibration parameters calculated from different software (internal and external orientation usually based on mathematical models and fixed chessboards);

- the appropriate data acquisition of the subject (parallel and/or convergent acquisition);

- the reconstruction algorithm used by the software for generating the dense cloud;

- the projection error in the scaling transformation.

A test was performed on the Facchino's Fountain in Rome with two different photogrammetric software applications; Agisoft Photoscan and 3D Zephyr. The same images and GCPs (5 points) were used. The first evaluation concerned the projecting error of the two numerical models. The MSE of Photoscan cloud is $3.5 \mathrm{~mm}$, while the Zephyr cloud is $3.1 \mathrm{~mm}$. This gap is mainly due to the choice of the points on the numerical models, characterized by different resolution. Although the error is quite the same, differences can be noticed in the dense cloud reconstruction. Measuring the distance between the two numerical models, in a threshold of $5 \mathrm{~mm}$, it is possible to highlight how the reconstruction algorithms determine also the final result (CloudCompare, Figure 3).

The histogram shows differences between the two point clouds generated by the corresponding software. Many discontinuities are evidenced along the entire area, both on the sculpture and the architectural part (Figure 4). Editing some settings (i.e. alignment and depth filtering) the position of the points in the virtual space can be improved, modifying also the final result.

Photomodeling systems generate not scaled numerical models. Two different approaches are possible for scaling the point clouds:

- direct measurements on the subject. This approach according to the authors is less accurate because it is based on direct distance between two points of the subject; the measurements depends also on the dimension of the subject and from the possibility to reach inaccessible parts;

- point coordinates. This system is based on the acquisition of specific points. Even though at least 3 points are enough to scale an object in virtual space, the scaling process needs almost 5-10 points well distributed on the cloud to reduce the projection error of the 3D model.

In archaeological context the integration between range-data and image-based systems is very useful for recognizing inaccessible parts and filling shadow areas produced from the laser scanner. Often point clouds generated from 
photomodeling systems have a higher resolution than those of the laser scanner. This difference in resolution could make the surface reconstruction and the representation difficult. Different tools offer the possibility to sub-sampling the clouds at the same resolution for a homogeneous result.

\section{REMOTELY PILOTED AIRCRAFT SYSTEM}

A particular attention has been dedicated to the Remotely Piloted Aircraft System (RPAS; drone) and the related digital photogrammetric acquisition. A complete research paper, published by Grussenmayer, evidenced interesting ideas and best practices for a correct approach both in the acquisition and processing phases with multi-copter rotary-wing type [14]. The paper presents also important information regarding the point clouds reconstruction and camera calibration.

Drones allow to fly in restricted areas and are very useful for the archaeological survey and photo interpretation, considering their capability to visualize from a different point of view archaeological environment (top view); most of them are equipped with a camera and a GPS antenna for controlling the flight.

Usually convergent data acquisition is suggested for satisfying dense-cloud reconstruction algorithms and to better calculate the extrinsic parameters of the camera. However, two are the main issues that influence the flight and the shot:

- the life of the batteries that currently have an autonomy of 20 minutes per flight;

- differences in image-texture homogeneity for each mission due to different radiometric values.

Usually photogrammetric software packages employ exif information for georeferencing the numerical model of the camera in the virtual space. The GPS antenna is not a differential system and its accuracy is between $1 \mathrm{~m}$ and $10 \mathrm{~m}$, depending from different factors. Despite the result seems to be accurate the integrated GPS increases the final projection error. Except where GPS data is corrected by a reference system, the model must be referenced with the aid of a total station or another GNSS system.

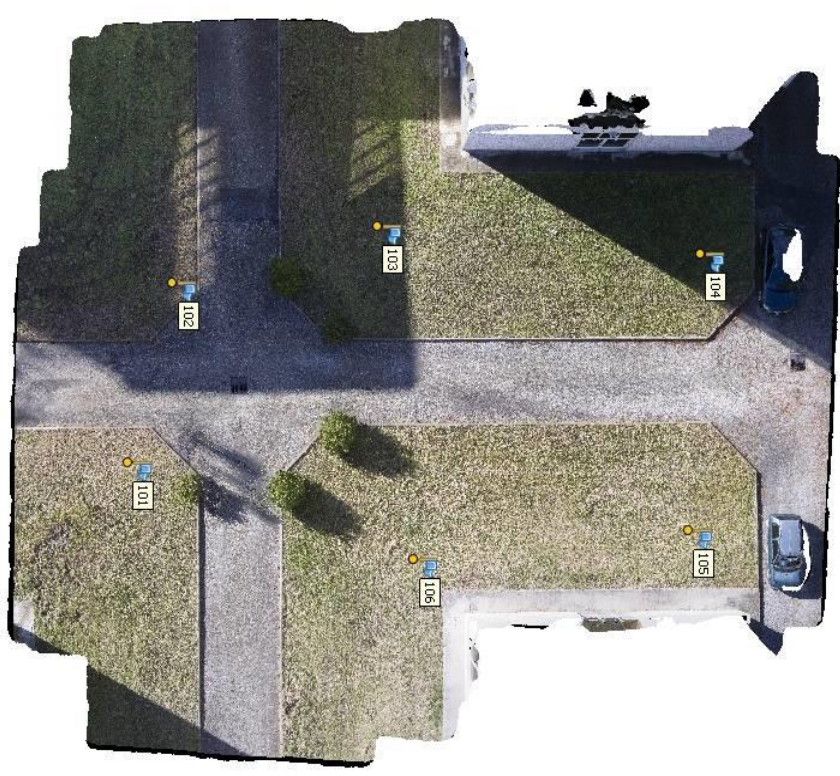

Figure 5. Ortho-rectified image of a flat surface improved with total station measurements.
A test was performed at $10 \mathrm{~m}$ high with 6 targets on the ground, measured both with a total station and directly on the numerical model generated by the photogrammetric software (Figure 5).

The starting measured distance between the target differs from the reality of about $0.60 \mathrm{~m}$. By orienting the numerical model in the software with local coordinates (in the Reference options) the problem can be partially solved, and the error decreased down to $0.045 \mathrm{~m}$. Another experimentation was performed with the same images without GPS data. This is possible disabling the reference accuracy in the alignment process or editing the exif file, removing GPS data. In the first case the external parameters of the cameras are calculated without GPS and the model is scaled. The second option generated an un-scaled dense cloud with a final error of 0.019 $\mathrm{m}$ (improved with a total station).

For the quick test a self-calibration method was used but in general a full calibration is advisable for avoiding distortions and improving the general accuracy of the project [15].

An important issue concerns the choice of the right height for taking photo. The resolution of the camera sensor today allows to generate ortho-rectified images with high details for any representation scale. However, in order to plan correctly a flight, it is important to establish the approximate height of the drone in relation to the representation scale. This is important to avoid excessive data and long processing time.

The GSD (Ground Sampling Distance) and the representation scale are the two important factors for choosing the drone height:

- The GSD represents the relationship between the sensor width of the camera (millimetres), the focal length (millimetres), the image width (pixel) and the flight height (meters);

- the representation scale generally used in archaeology for a survey is 1:100, 1:50 and 1:20 for details.

GSD was theorized for a DJI Phantom 4. The drone has a width sensor of $6.17 \mathrm{~mm}$, a focal length of $3.61 \mathrm{~mm}$ and an image width of 4000 pixel. At a height of $10 \mathrm{~m}$ the GSD is 0.43 $\mathrm{cm} /$ pixel, a value more accurate than the one required. By defining the GSD in relation to the supposed representation scale, it is possible to provide the approximate height for the drone flight.

Supposing a linear dimension of the pixel of $1.5 \mathrm{~cm}$, the drone should fly at $35 \mathrm{~m}$ height (presuming that the resolution of the result cannot be lower than the error allowed in the 1:50 representation scale). Certainly, this represents only a theoretical approach because different factors can influence the choice of the height. For instance, the extension of the area can determine the best choice above all when the excavation is not particularly extensive.

The GSD calculated on ortho-rectified images is an average value that depends also on the geomorphology of the ground. The flight should be done maintaining always the same height from the ground. The right height of the drone allows to acquire a limited number of images for a large territory, optimizing the energy of the batteries.

A further consideration concerns the relationship between the rectified image and $2 \mathrm{D}$ representation, normally executed by drawing the main archaeological features and details. The archaeologist should evidence a number of details that satisfy the chosen representation scale (otherwise it is useless to have high-resolution ortho-photos). The drawing is the only method 


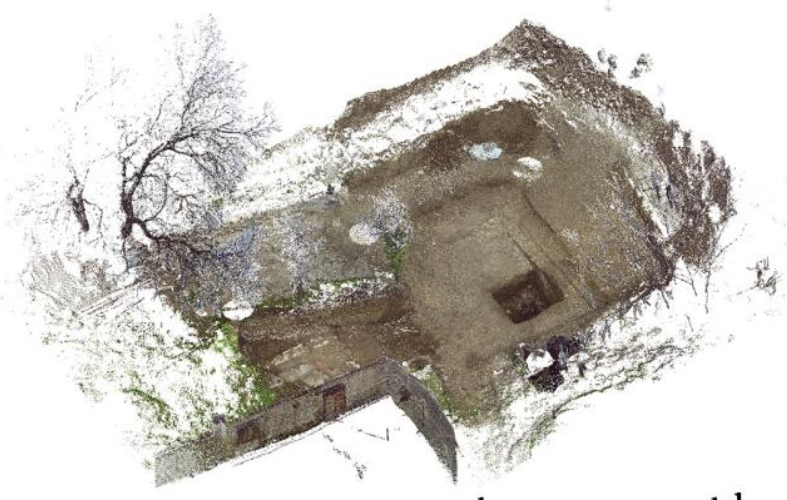

laser scanner model

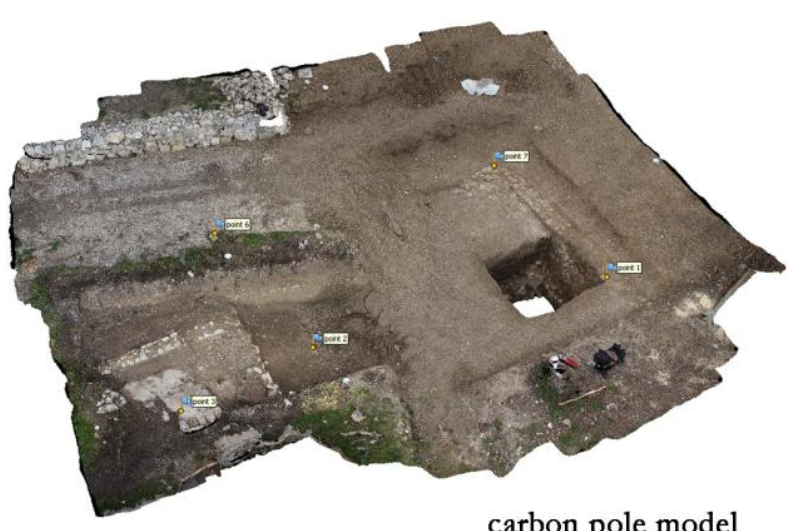

carbon pole model

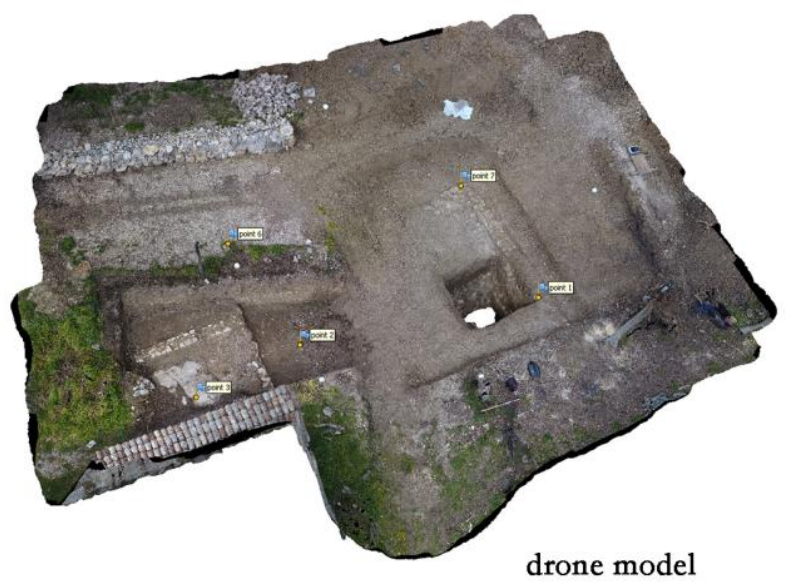

Figure 6. Three numerical models of an archaeological excavation. The test was performed for highlighting differences between laser scanner data and digital photogrammetry from a drone and a carbon pole.

based on critic approach and able to give information useful for the historical reconstruction of a monument.

Recently an innovative method for the representation of rupestrian architecture was experimented and adopted for the archaeological representation [16]. The method is based on the use of the "contour lines" for describing objectively all forms not attributable to a defined geometry, such the rock-hewn architecture. This representation method can be adopted also for archaeological excavations where structures and layers were altered by the time. The system allows to define the edges and the outline that must be represented in the chosen representation scale, modifying the number of the sections on the numerical models with specific settings.

\subsection{Carbon Pole Vs Drone}

A specific experimentation was tested for comparing the acquisition and processing steps between a camera mounted on a carbon pole and the built-in camera of the drone on the same archaeological area (Figure 6). The comparison was planned in order to evaluate the impact of a proper exploitation of different technologies in order to reduce errors.

For the test a carbon pole was used with a maximum extension of $6 \mathrm{~m}$ (Fanotec carbon pole, Figure 7). This configuration is the only able to support a reflex in inclined position due to the weight of the camera. The camera used was a Canon 5D Mark II with a $28 \mathrm{~mm}$ optic lens. Another configuration is available with an extension of $9 \mathrm{~m}$, but no reflex cameras can be mounted.

The comparison was executed with a semi-professional rotary-wing platform (DJI Phantom 4) with a built-in camera (FC330).

An archaeological excavation was investigated for the experimentation, characterized by different features like ancient structures and mosaics but also some obstacles such trees and a little building for an overall area of $500 \mathrm{~m}^{2}$. The experimentations aimed mainly at:

- defining an acquisition procedure repeatable also in other contexts;

- defining some aspects concerning both the acquisition and the processing phases;

- $\quad$ understanding which survey techniques are suitable in archaeological excavations considering also the issues related to the environment.

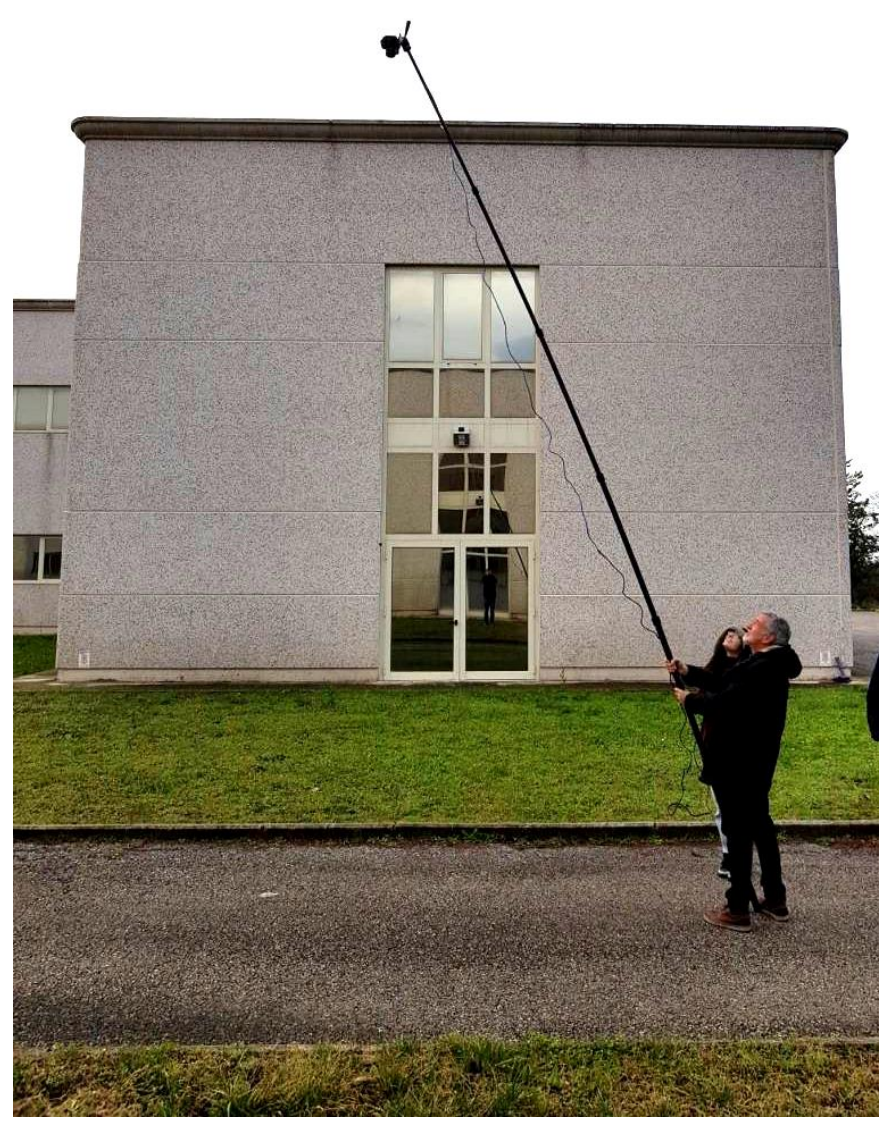

Figure 7. Fanotec carbon pole used for testing the GSD of a reflex camera at different heights. 

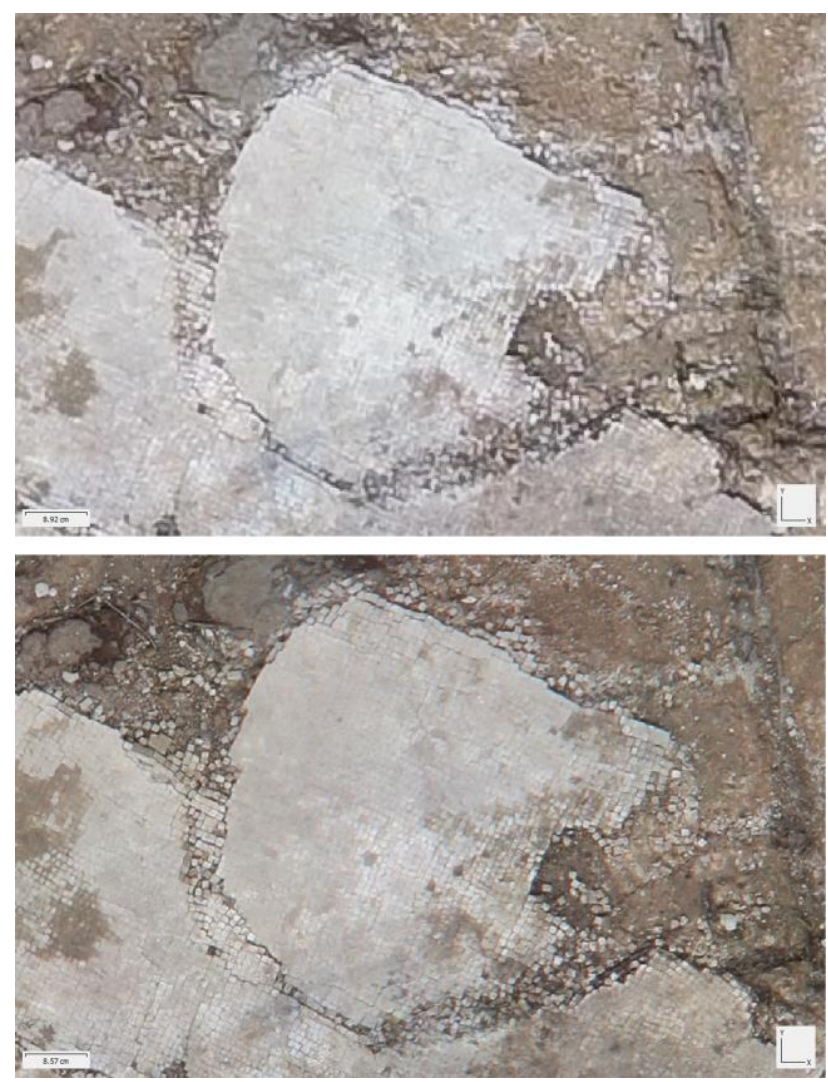

Figure 8. Orthomosaics of the archaeological area; the first image, from the drone, has a resolution of $2.5 \mathrm{~mm} /$ pixel while the second image, from the carbon pole camera, has a resolution of $1.1 \mathrm{~mm} / \mathrm{pixel}$.

As known photomodeling generates non-metric numerical models that need to be scaled with measurements or coordinates. For the test 8 scans were also acquired and matched together in the same reference system. In this way the choice of the points for scaling the clouds in the processing step has been possible. Information regarding the two acquisitions is reported below:

- the acquisition with the drone was performed in GPS mode at 7-8 $\mathrm{m}$ from the ground (the height normally is referred to the starting point). A parallel/convergent acquisition was performed for the entire area even if some problems occurred near the trees. Due to the weak GPS signal the drone was switched in manual mode (ATTI mode) with high difficulties in the management of the aircraft, subject also to the wind. For that reason, a part of the excavation was acquired at different height and with tilted camera for avoiding the closeness with the trees. The average GSD of the images is $0.30 \mathrm{~cm} /$ pixel at $7 \mathrm{~m}$ and the theoretical area covered for each image is $108 \mathrm{~m}^{2}$;

- $\quad$ in the acquisition step the carbon pole was inclined at 60-65 degrees to avoid the shadows and to visualize only the archaeological area. For shooting images, a sampling of one photo each 3 steps was used. It would be possible to mount a tablet on the pole to visualize the survey area; however, the light radiation would make it difficult and inconvenient. The acquisition method was organized for avoiding footprints on the ground and shadows, considering the ease of moving the earth during the excavation. The starting point depended from the position of the sun. Parallel acquisitions were performed at a height of $5.20 \mathrm{~m}$. This value was supposed and calculated exploiting the following trigonometric formula:

$b=l \sin \beta$

where $h$ is the height of the camera, $l$ is the length of the pole $(6 \mathrm{~m}), \sin \beta$ is the inclination of the pole $\left(60^{\circ}\right)$. At that height the average GSD of the images is 0.12 $\mathrm{cm} /$ pixel, but the covered area is only $28 \mathrm{~m}^{2}$ for each image. A total of 70 images was acquired over the entire area.

Some difficulties were noticed in the management of the carbon pole and its weight, beyond the difficulties of moving on the excavation, depended mainly from the morphology of the area.

The processing step was performed with Photoscan and JRC Reconstructor. The laser scanner clouds were registered together for achieving local coordinates of the points for the scaling process.

For the alignment and dense cloud reconstruction of the two groups same parameters were set (High Alignment and Medium Dense Cloud).

Two different clouds were generated, one from the carbon pole (a) and another from the drone (b) without GPS data.

From the laser scanner 6 points were identified for scaling the photogrammetric models. The model from the carbon pole (a) registered an error of $7 \mathrm{~mm}$ on the ground, the second model without the GPS (b) registered an error of $8 \mathrm{~mm}$.

The comparison made in CloudCompare evidenced a good overlapping between the models a and $\mathrm{b}$ with little mismatching in the reconstruction process.

The results of the experimentation can be summarized as follow:

- the models were generated with different number of points; in particular, model (a) is composed by a number of points 3 times higher than the model (b).

- after the scaling process both the models are enough accurate for the archaeological representation. Little differences are not evident in the 1:50 representation scale;

- $\quad$ model (b) covered an area greater than model (a);

- the drone is simple to use and can cover large area in about 20 minutes (thanks to the long-life batteries). The main problems regarded the obstacles and the built-in camera quality that is not comparable with a reflex camera;

- the carbon pole is a very suitable instrument for the photomodeling acquisition on the excavation. By using a wide lens, such the $28 \mathrm{~mm}$, it is possible to acquire a lot of data in very short time, exploiting the properties and the quality of a reflex camera. No permission from the civil aviation are necessary for using a pole in an excavation. The major difficulty regarded the final weight of the device used for long time. The resolution and the quality of the results are evident in the final step even if the processing time has taken more time;

- the problem of the image homogeneity was influenced by acquisition time; with the carbon pole the acquisition needs 25 minutes while the multi-copter employed only 10 minutes for the entire area. The test was performed during a cloudy day for avoiding high contrast on the ground, but sometime the acquisition stopped due to the direct light radiation. 


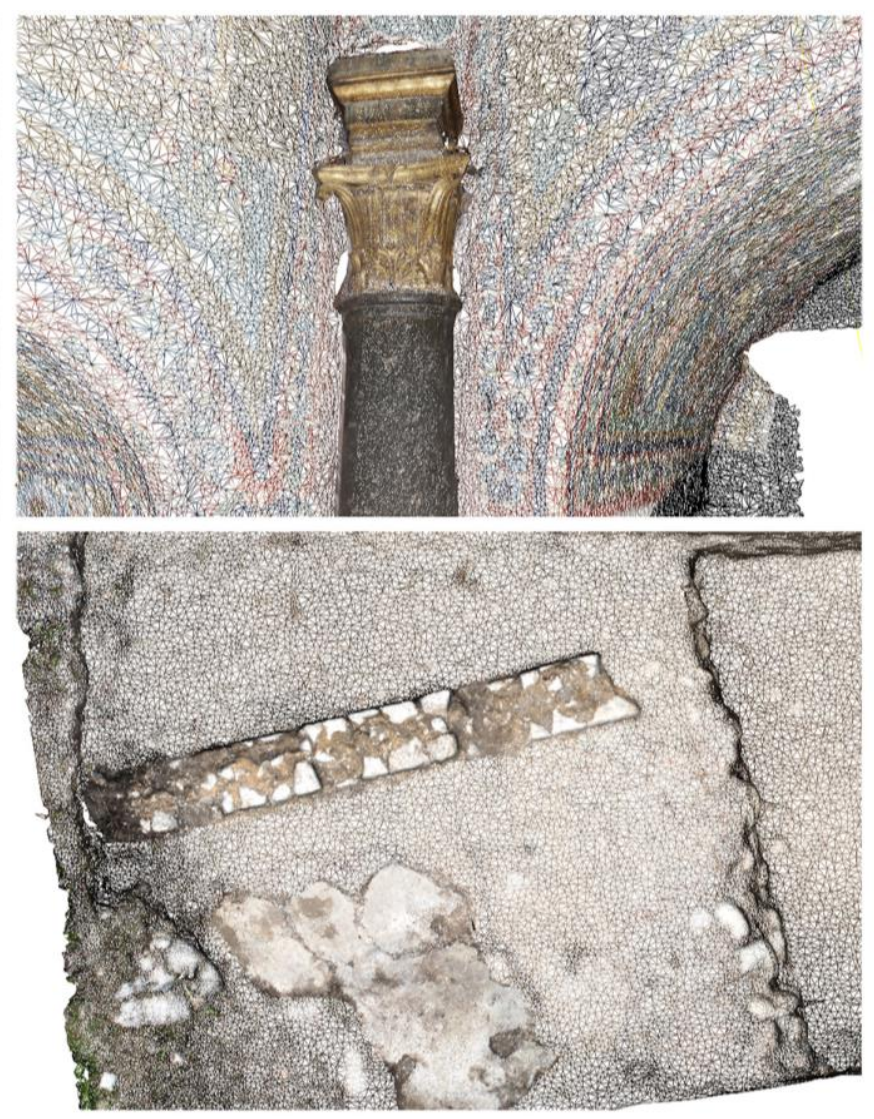

Figure 9. Examples of different surface reconstructions based on controlled multi-resolution methods.

The last comparison regarded the quality of the orthomosaics that represent the base for the digitalization in the archaeological representation. Differences are present above all for the details (mosaics, Figure 8) even if the representation scale allows to use indifferently both the results.

\section{THE SURFACE RECONSTRUCTION}

Once point clouds have been correctly filtered (noise reduction and pre-processing filters), the surface reconstruction process becomes necessary to achieve the final 3D representation. Surface reconstruction must be considered as a further interpolation of the data.

This approximation of the surfaces depends mainly on the algorithms used for the reconstruction but also from the critical approach to the subject. To obtain good results, data synthesis and critical approach must be performed from the archaeologists, defining which are the important parts and the representative points of the $3 \mathrm{D}$ model.

In this step points are filtered for the representation of the subject with different level of details [17]. The process is executed through the meshing techniques. This transformation is necessary to reduce the point clouds, to create connections among the points, to measure the connected and continued surfaces and to apply different maps from the 2D images (texture mapping process).

Different mesh generators for unstructured clouds exist, even if the most common are mainly based on the studies made by Owen in 1998 [18]. The most used method for generating triangular meshes is that known as Delaunay criterion. This is therefore not just a real algorithm, but a selection criterion associated to an algorithm, which subsequently generates the triangular surfaces [19].

The meshing technique is an automated procedure that does not allow the full control of the interpolation, even if some of the algorithms present editable values (i.e. number of triangles, ratio, accuracy, size). Independently from the algorithm used, the surface reconstruction aims at generating light numerical models with high details.

The main question concerns the choice of the number of triangles, a value difficult to manage in the reality. The issue is how many triangles are necessary for a detailed surface reconstruction, considering that in the representation discipline we are not used to thinking about triangles as units of measure. Most of the software applications are able to perform different algorithms for automatic processes with reliable results. The problem is given by the target of the research and the expected results.

The possibilities for organizing a surface reconstruction, independently from the algorithm used, are four:

- a low-res numerical model, poor of details but easy to manage in the virtual space;

- a high-res numerical model, high in details but difficult to manage with the memory of the computer, characterized by the entire point cloud;

- a multi-resolution model based on automated algorithms, able to recognize and fit discontinuities on the model (commonly used);

- a multi-resolution model based on "semantic" subdivision of the point cloud, applying suitable meshing parameters for each architectonic or sculptural element (Figure 9). A point cloud can be divided in different entities such as the main walls, the columns, the capitals and altars, separating the general geometry of the structure from the architectural details. For instance, the main walls need low resolution while the columns, the capitals and the altar need a large number of triangles [20].

The result should be a light numerical model with a good number of details (semi-automatic process).

The surface model can be also improved through:

- the optimization methods, such as the smoothing algorithms that maintain the connectivity but rearrange the nodes of the triangles;

- the cleanup algorithms that maintain the position of the nodes but change their connectivity. The latter improves the general quality of the mesh and their organization but contemporary generates different interpolations that alter original data.

Other procedures are used to improve the final result:

- the repair of the mesh that is required when the algorithmic operation is not completely successful, so the model can have holes, or topological problems (i.e. self intersections);

- the decimation filter that uses a series of algorithms to simplify the model and generate multi-resolution models;

- the densification or refinement processes to increase the detail of the mesh. The algorithms for the densification processes (such as Edge bisection, Point Insertion, Templates) are countless.

The archaeological representation scale is able to hide differences between the position of the points and the related 
triangle. Sometimes this problem is completely irrelevant for the representation, considering the large amount of data. The suggestion is to systematize the models according to a precise organization of the data decided from the user a-priori and considering the result expected for the research.

\section{THE TEXTURE MAPPING PROCESS}

The surface reconstruction is also finalized to the texture mapping process. The aim is to map high resolution images on unstructured surface model in order to generate a reality-based virtual model.

Also in this case it is necessary to take images at high resolution and with a quality optic lens. Usually a $28 \mathrm{~mm}$ lens is a good compromise between shooting range and radial distortions. The shape and the details of the archaeological subject influence the entire shot. The most common software packages use similar systems for performing texture mapping process, however a test has been conducted with the aid of $J R C$ Reconstructor, for the closeness to the descriptive geometry issues.

The pipeline can be summarized in the two following steps: the full camera calibration;

the image projection process on the numerical model (mesh).

The full camera calibration is performed between the image and the point cloud. The accuracy of this operation depends on the optic lens used, the number of homologous points identified and their geometric arrangement, besides the different resolution of the point clouds and images (Figure 10). Recognizable homologous points can be described with a different number of pixels (for instance, the eye of a holy character). When the resolution of the images differs far (greater/lower) from that of the numerical model used by the laser, the projection error increases.

Despite 11 points are necessary for the full camera calibration, the authors suggest more than 20 points per image, considering the complexity of an investigated area and the level of detail (LoD). The mathematical models are also able to rectify wide lenses such as a $14 \mathrm{~mm}$, but it requires the use of more than 25 points per image (increasing the minimum sensibility requested for solving parameters). To determine the external parameters of an image that represents an architecture, the suggestion is to choose the correspondences on the representative points of the architecture, facilitating the image projection process.

A particular difficulty occurs during the recognition of the homologous points. The choice of the $3 \mathrm{D}$ points is done on the

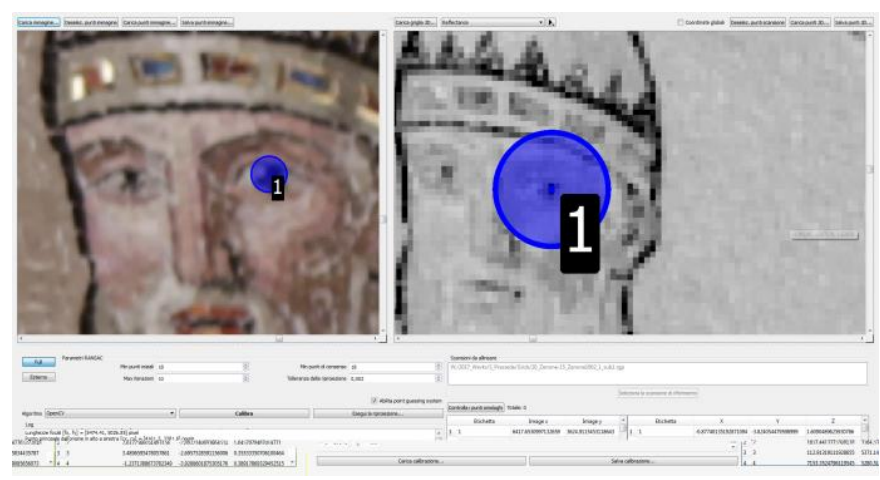

Figure 10. The different resolution between laser scanner and the related image can generate a high projection error in the full calibration process. equirectangular projection of the point cloud (or directly on the 3D model). In this projection, only the central area is useful for the identification of the points, while in the polar areas it is impossible to see details due to their deformation. The possible solutions of the problem are two:

the transformation of equirectangular projection into cubic projection, with the support of specific software for the recognition of the correspondences;

- the acquisition step made in different way (tilting the camera) considering also the poles deformation above all in the indoor spaces.

The authors experimented also an innovative procedure with the use of "Virtual Scan" tool by Gexcel [21, 22]. The tool is able to collect virtual scan of a subject in virtual space by using different projection systems such as orthographic, cylindrical, spherical and perspective cameras in any virtual position, established by the user. For instance, by setting a spherical camera, the tool allows to register a new equirectangular grid $(360 \times 180)$, composed by the same points without altering their position (graphics card assembled on the computer can influence the resolution and reduce the processing time). A perspective camera has been set with the projection centre directed to the roof and with a wide FOV (Field of View, $120^{\circ}$ ). A new structured scan has been collected for camera calibration process without any deformation in the $2 \mathrm{D}$ structured image.

The full calibration was repeated for all the images and for each calibration a new projector was created. The origin of the projector coincides with the coordinate of virtual projection centre of the related image $\left(\mathrm{X}_{0}, \mathrm{Y}_{0}, \mathrm{Z}_{0}\right)$. By turning it on and off (loading the image) it is possible to view the projected image on the model and to evaluate the accuracy of the process, especially on the edges and corners of the subject (Figure 11).

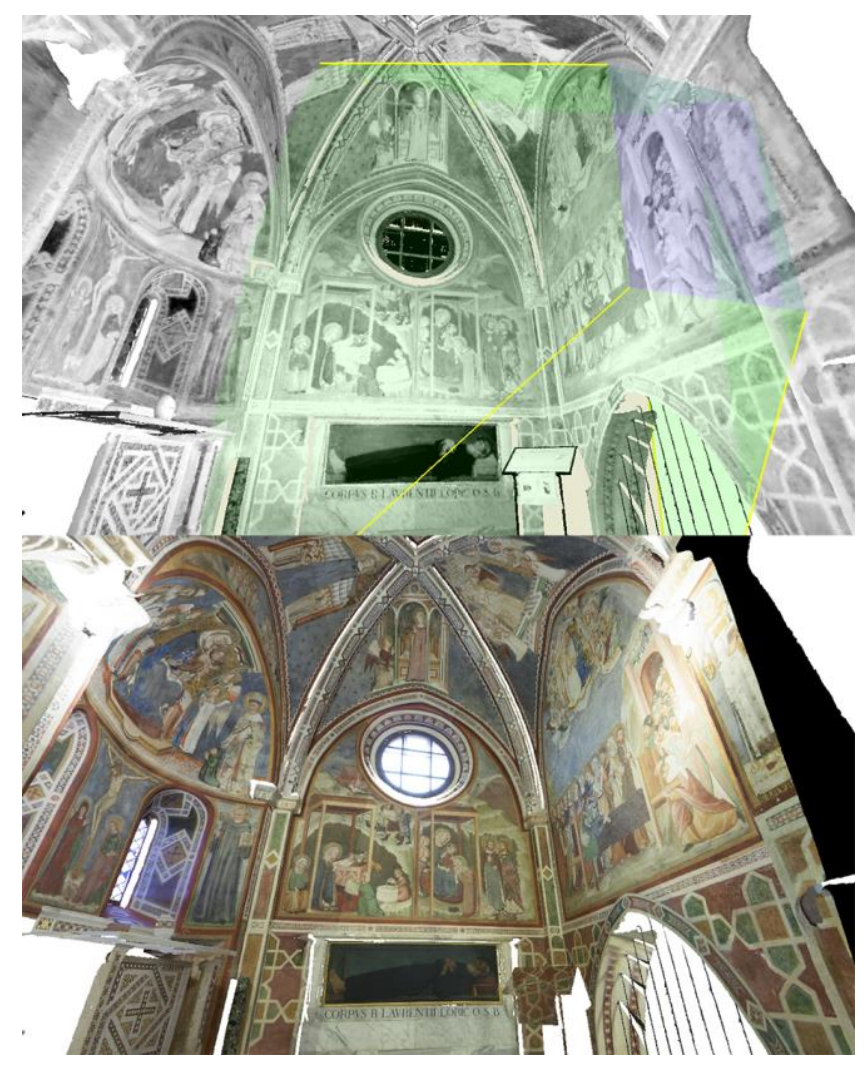

Figure 11. The texture mapping process increases the quality of the result of an architecture even if the main projection errors can occur in the edges and corners of the numerical model. 
For that reason, it is important to have a light model with high details for evidencing mismatching between the projected images and the structure of the numerical model. By lighting on multiple projectors some errors can be noticed, above al comparing the main features of the images. In order to reduce the mismatching, the images in the overlapping areas can be cut or, where possible, the same points used in the full calibration step can be detected.

Alternatively, the tool allows to set the boundaries and orientation of the image to be projected on the model. In this way the points recognition is facilitated during the identification process, exploiting the entire image for solving the internal parameters [23].

Once the error (pixel) allows to visualize the homogeneous distribution of the image projections (no splitting images), texture mapping process can be performed directly on the mesh model. Usually a multi-blending algorithm is used in order to correct the difference in colour tone. The result is a realitybased virtual model able to describe the complexity of an archaeological subject from different points of view.

\section{CONCLUSION}

Some final considerations are necessary for fixing the main objectives of the paper. The authors aimed at giving a general view on the survey issues in Archaeology, evidencing two important elements:

- the importance of the new technologies as support to archaeological research;

- the innovative investigation methods and the risk to produce new errors in the entire survey process.

In the last 20 years, surveying activity has changed in the archeological excavations, so that today it is very important to be aware on the use of these techniques.

Compared to the past the acquisition and processing phases are more complex, considering the amount of data and the operations necessary to accomplish an accurate result. These technologies require more knowledge borrowed from different disciplines and application fields such as the Descriptive Geometry, the Computer Graphics, the Drawing, the Photography, the theory of the errors and many others. All these elements have become for the archaeologists innovative tools that, if well used, are able to generate new information useful for the investigation and study of an archaeological subject.

On the other hand, the technologies must not divert attention from archaeological issues and should be aimed at solving different historical and methodological questions, such the phases of a monument or innovative representation methods.

A numerical model represents only the base for further processes. A reality-based virtual model can be managed in different ways in order to create new forms of representation difficult to reproduce with the traditional methods.

The test presented in the paper showed how to manage technologies in order to reduce as much as possible the errors in the different phases of the survey pipeline.

\section{ACKNOWLEDGEMENTS}

The paper is the result of the collaboration between the authors. In particular, Andrea Angelini developed the paragraphs 1, 2 and 5, Damiano Portarena the paragraphs 3, 4 and 6.

\section{REFERENCES}

[1] A. Angelini, D. Portarena, Some considerations on measurement errors in archaeological survey, in IMEKO International Conference on Metrology for Archaeology and Cultural Heritage Lecce, Italy, October 23-25, 2017, pp. 541-546.

[2] G. Vosselman, H. G. Maas, Airborne and Terrestrial Laser Scanning, Whittles, Caithness, UK, 2010, ISBN 978-1904445-87-6.

[3] F. Remondino, S. Campana, ed., 3D Recording and Modelling in Archaeology and Cultural Heritage - Theory and best practices, BAR International Series 2598, Oxford, 2014, ISBN 978-14-0731230-9.

[4] M. Docci, D. Maestri, Manuale di rilevamento architettonico e urbano, Editori Laterza, Roma, 2012, ISBN 978-88-420-9068-7.

[5] W. Boehler, M. Bordas Vincent, A. Marbs, Investigating laser scanner accuracy, in ISPRS archives, vol. XXXIV, Part5/c15, Antalya, 2003, pp. $696-701$.

[6] F. Remondino, Heritage Recording and 3D Modeling with Photogrammetry and 3D Scanning, Journal on Remote Sensing, 3, 2011, pp. 1104-1138.

[7] M. Carpiceci, Modelli geometrici e costruzioni grafiche per il rilevamento architettonico. Idee e proposte per una migliore gestione dei dati grafici e numerici nel rilevamento architettonico, Aracne, Roma, 2012, ISBN 978-88-548-5153-5.

[8] P. J. Besl, N. D. McKay, A Method for Registration of 3-D Shapes, IEEE Transactions on Pattern Analysis and Machine Intelligence, Vol. 14 (2), 1992, pp. 239-256.

[9] S. Rusinkiewicz, M. Levoy, Efficient variants of the ICP algorithm, in IEEE Proceedings of Third International Conference on 3-D Digital Imaging and Modeling, Quebec City, 2001, pp. 145-152.

[10] S. Campana, M. Forte, ed., Remote Sensing in Archaeology. XI Ciclo di Lezioni sulla Ricerca applicata in Archeologia (Certosa di Pontignano 1999), All'Insegna del Giglio, Firenze, 2001, pp. 329354. ISBN 88-7814-175-5.

[11] C. D. Ghilani, P. R. Wolf, Elementary Surveying. An introduction to geomatics, Prentice Hall, New Jersey, 2012, pp. 191-275.

[12] E. M. Mikhail, J. S. Bethel, J. C. McGlone, Introduction to Modern Photogrammetry, John Wiley \& Sons, USA, 2001. ISBN 978-0-471-30924-6.

[13] F. Remondino, M. G. Spera, E. Nocerino, F. Menna, F. Nex, State of the art in high density image matching, The Photogrammetric Record, Vol. 29, Issue 146, June 2014, pp. 144-166.

[14] A. Murtiyoso, P. Grussenmeyer, Documentation of heritage buildings using Close-range uav images: dense matching issues, comparison and case studies, The Photogrammetric Record, 32(159), 2017, pp. 206-229.

[15] T. Schenk, Introduction to Photogrammetry. Department of Civil and Environmental Engineering and Geodetic Science, The Ohio State University, USA, 2005.

[16] M. Carpiceci, C. Inglese, Laser Scanning and Automated Photogrammetry for the knowledge and the representation of the architecture cave in Cappadocia: Sahinefendi and the Open Air Museum in Goreme, In CAA 2014: 21 ${ }^{\text {st }}$ Century Archaeology Concepts, methods and tool. Proceedings of the $42^{\text {nd }}$ annual conference on Computer Applications and quantitative methods in Archaeology, Paris, France, 2015, pp. 87-94.

[17] C. Bianchini, Documentation of Mediterranean ancient theatres. Athena's activities in Mérida, Gangemi Editore, Roma, 2012, ISBN 978-88-492-2524-2.

[18] S. J. Owen, A Survey of Unstructured Mesh Generation Technology, Proc. of $7^{\text {th }}$ International Meshing Roundtable, Oct. 26-28, 1998, Dearborn, Michigan, USA, pp. 239-267.

[19] R. Migliari, Geometria Descrittiva. Tecniche e Applicazioni, Vol. II, 2009, ISBN 978-88-251-7330-7.

[20] M. Carpiceci, M. Russo, A. Angelini, The digital model of the S. Zenone chapel inside Santa Prassede in Rome. A case study for the morphological analysis, in 23rd International Conference on 
Virtual Systems and Multimedia (VSMM2017): Through the Looking Glass - Back to the Future of Virtual Reality, Dublin, 2017, pp. 238-246.

[21] M. Sgrenzaroli, G. P. M. Vassena, Tecniche di rilevamento tridimensionale tramite laser scanner. Volume 1 - Introduzione generale, Starrylink Editrice, Brescia, 2007, ISBN 978-88-8972073-8.
[22] A. Angelini, G. Capriotti Vittozzi, M. Baldi, The high official Harkhuf and the inscriptions of his tomb in Aswan (Egypt). An integrated methodological approach, in S. Grassini, A. Santoriello, (eds) The e-Journal of the International Measurement Confederation (IMEKO) Acta IMEKO, Vol. 5 (2016) 2, pp. 71-79.

[23] S. Dequal, Un concetto innovativo: l'immagine solida. Bollettino SIFET, 1/2003, pp. 67-76. 